\title{
Scalar Nanosecond Pulse Generation in a Nanotube Mode-Locked Environmentally Stable Fiber Laser
}

\author{
R. I. Woodward, E. J. R. Kelleher, D. Popa, T. Hasan, F. Bonaccorso, \\ A. C. Ferrari, S. V. Popov, and J. R. Taylor
}

\begin{abstract}
We report an environmentally stable nanotube mode-locked fiber laser producing linearly-polarized, nanosecond pulses. A simple all-polarization-maintaining fiber ring cavity is used, including $300 \mathrm{~m}$ of highly nonlinear fiber to elongate the cavity and increase intracavity dispersion and nonlinearity. The laser generates scalar pulses with a duration of $1.23 \mathrm{~ns}$ at a center wavelength of $1042 \mathrm{~nm}$, with $1.3-\mathrm{nm}$ bandwidth and at $641-\mathrm{kHz}$ repetition rate. Despite the long cavity, the output characteristics show no significant variation when the cavity is perturbed, and the degree of polarization remains at $97 \%$.
\end{abstract}

Index Terms-Laser mode locking, fiber lasers, optical polarization, optical pulse shaping.

\section{INTRODUCTION}

$\mathbf{P}$ ASSIVELY mode-locked fiber lasers have become established tools for generating picosecond and femtosecond pulses due to their compact design, alignment-free waveguide format and low cost [1]. These advantages over their solid-state counterparts have driven interest in extending the modelocked fiber laser format to produce highly-stable nanosecondduration pulses at sub-megahertz repetition rates [2]-[6]. Such pulse sources could simplify amplification schemes [2] and enable lower threshold-power supercontinuum generation [7].

Mode-locked pulse characteristics are determined by the laser cavity dispersion map [8], [9]. In particular, all-normal dispersion cavities have been reported to produce chirped pulses with energies up to hundreds of nanojoules [10]-[12]. These pulses have been reported as examples of dissipative solitons [8], which are encountered in a wide range of physical systems [9]. In mode-locked lasers, the dissipative soliton formation mechanism requires a balance between gain and intensity-dependent loss, in addition to interplay between dispersion and nonlinearity [9]. Spectral filtering is also required

Manuscript received April 8, 2014; revised May 13, 2014; accepted June 2, 2014. Date of publication June 12, 2014; date of current version July 25, 2014 This work was supported in part by the Royal Society Wolfson Research Merit Awards, in part by the European Research Council through the NANOPOTS, Hetero2D, European Union Graphene Flagship under Contract 604391, in part by the Engineering and Physical Sciences Research Council under Grant EP/K01711X/1, Grant EP/K017144/1, and Grant EP/L016087/1, in part by Emmanuel College, Cambridge, and in part by a Newton International Fellowship.

R. I. Woodward, E. J. R. Kelleher, S. V. Popov, and J. R. Taylor are with the Femtosecond Optics Group, Department of Physics, Imperial College London, London SW7 2BW, U.K. (e-mail: r.woodward12@ imperial.ac.uk; edmund.kelleher08@imperial.ac.uk; s.popov@imperial.ac.uk; jr.taylor@imperial.ac.uk).

D. Popa, T. Hasan, and A. C. Ferrari are with the Cambridge Graphene Centre, University of Cambridge, Cambridge CB3 OFA, U.K. (e-mail: dp387@cam.ac.uk; th270@cam.ac.uk; acf26@cam.ac.uk).

F. Bonaccorso is with the Cambridge Graphene Centre, University of Cambridge, Cambridge CB3 OFA, U.K., and also with the Istituto Italiano di Tecnologia, Genova 16163, Italy (e-mail: fb296@cam.ac.uk).

Color versions of one or more of the figures in this letter are available online at http://ieeexplore.ieee.org.

Digital Object Identifier 10.1109/LPT.2014.2330739 to stabilize the pulse, often in the form of an intracavity filter [8] or arising from the limited gain bandwidth of the system [6]. More recently, this concept has been extended by elongating cavities up to kilometer scales, giving very large intracavity dispersion to produce nanosecond duration pulses [3]-[5]. Chirped fiber Bragg gratings have also been used to provide a large lumped dispersion to generate nanosecond pulses at megahertz repetition frequencies [13], [14]. Whilst it should be noted that lasers of this nature can also operate in a noisy regime producing periodic bursts of incoherent noise-like pulses [3], with appropriate cavity design and adjustment, coherent pulses can be produced with a giant linear chirp [5]. Such pulses could be directly amplified in a chirped pulse amplification scheme to avoid pulse distortion during power scaling, removing the need for a stretcher stage [1] and the linear chirp suggests compression is possible [5]. The low-repetition rate of long-cavity lasers enables higher pulse energies to be achieved, which could reduce the pump power required for supercontinuum generation, with applications in fluorescence imaging and broadband spectroscopy [7], [15].

The sensitivity of optical fibers to environmental stresses can, however, limit practical application of long-cavity mode-locked fiber lasers. Such disturbances can alter the fiber birefringence, which can unpredictably degrade laser performance [16]. Additionally, nanosecond modelocked pulse sources reported to date produce vector pulses [3]-[5], [13], [14], i.e. pulses consisting of two coupled propagation modes along orthogonal polarization axes [16]. These modes can exchange energy as the birefringence changes, causing the polarization state to evolve unpredictably over time [16]. When both group and phase velocities are equal along orthogonal polarization axes, i.e. polarization-locked vector solitons [17], the pulse propagates without evolution in its polarization state [17], [18]. A scalar pulse (i.e. pulse with a single polarization component [16]), with a fixed linear polarization state is preferred for many applications where polarization-dependent changes in the material are important, such as biological imaging [19] and micromachining [20].

Environmental stability of all-fiber lasers can be improved by using polarization-maintaining (PM) fibers [16]. PM fibers reduce the coupling efficiency between polarization modes, minimizing the influence of disturbance-induced birefringence changes [1], [16]. An intracavity polarization-selective element can also be added, so the optical field in one polarization axis dominates, leading to linearly-polarized light propagation. To date, all-PM cavities producing broad chirped pulses in the allnormal dispersion regime have been demonstrated using both a nonlinear amplifying loop mirror [21] and a semiconductor 
saturable absorber mirror (SESAM) [22] to initiate modelocking, producing 68 ps [21] and 200ps [22] pulses, respectively. The degree of polarization (DOP), i.e. the polarized portion of the beam, was not measured in [21] and [22], and nanosecond scalar pulse formation in the normal dispersion regime has, until now, not been confirmed.

Carbon nanotubes (CNTs) and graphene have emerged as promising saturable absorbers (SAs) [23]-[25] with ultrafast recovery time [26], [27], the ability to support short pulses [28], [29], and with a number of favorable properties, such as broadband operation [30], and ease of fabrication [24] and integration [24] into all-fiber configurations [29], [31]. Broadband operation is an intrinsic property of graphene [32], while in CNTs this can be achieved using a distribution of tube diameters [30]. A variety of techniques have been implemented in order to integrate CNTs and graphene into lasers [33]. E.g., graphene can be integrated in various optical components [33], [34], with the possibility of controlling the modulation depth [34]. CNTs and graphene can also be embedded in polymer matrices that can easily be integrated into fiber lasers [33]. CNT composites can exhibit large modulation depths [29], [30], which are preferred for fiber lasers [1].

Here we report scalar nanosecond pulse generation in an environmentally stable mode-locked laser. We use an all-fiber all-PM ring cavity incorporating a CNT-SA composite to initiate mode-locking. We measure the output polarization properties to confirm scalar dissipative soliton operation, with a linear polarization state. Laser stability and energy fluctuations are also measured to illustrate the robustness of the pulse source.

\section{ExPerimental Setup}

To match the operation wavelength of Yb-doped fiber lasers $(\sim 1060 \mathrm{~nm}), \quad$ CNTs with $\sim 0.8 \mathrm{~nm}$ diameter are required [35], [36]. We use CNTs produced by Catalytic Chemical Vapor Deposition (CCVD) of $\mathrm{CH}_{4}$ over $\mathrm{Mg}_{1-x} \mathrm{Co}_{x} \mathrm{O}$ solid solution containing Mo oxide [37]. The absorption spectrum of the CNT powder is shown in Fig. 1 (black line) with a peak between 1030 and $1190 \mathrm{~nm}$. The desired operating wavelength is also highlighted (red dashes).

To further investigate the tube diameter distribution, before and after composite fabrication, and monitor defects, we measure the Raman spectra of the purified powder at $457 \mathrm{~nm}$ $(2.71 \mathrm{eV}), 514.5 \mathrm{~nm}(2.41 \mathrm{eV})$, and $632.8 \mathrm{~nm}(1.96 \mathrm{eV})$. In the low frequency region, the Radial Breathing Modes (RBMs) are observed [38]-[40]. Their position is inversely related to the diameter, $d$ [38]-[40] by $\operatorname{Pos}(\mathrm{RBM})=\mathrm{C}_{1} / \mathrm{d}+\mathrm{C}_{2}$. We use $\mathrm{C}_{1}=214.4 \mathrm{~cm}^{-1}$ and $\mathrm{C}_{2}=18.7 \mathrm{~cm}^{-1}$. A variety of $\mathrm{C}_{1}$ and $\mathrm{C}_{2}$ have been proposed in literature, see [39], [40]. Here we use $\mathrm{C}_{1}=214.4 \mathrm{~cm}^{-1}$ and $\mathrm{C}_{2}=18.7 \mathrm{~cm}^{-1}$ [41], derived in [38] by plotting the resonance energy as a function of $\operatorname{Pos}(\mathrm{RBM})^{-1}$ without any additional assumptions. The RBM spectra of the powders [Fig. 1(b)] (black lines) show a narrow distribution, spanning the $140-377 \mathrm{~cm}^{-1}$ range. This corresponds to $\sim 0.6-1.8 \mathrm{~nm}$ diameter. Fig. 1(c) plots the spectra in the $\mathrm{G}$ region of purified CNTs (black curves). A weak $\mathrm{D}$ band is observed $[\mathrm{I}(\mathrm{D}) / \mathrm{I}(\mathrm{G})=0.04]$, indicating small number of defects [42], [43]. A polymer composite is then fabricated via solution processing. Fig. 1(a) plots the absorption spectra of the PVA (grey line), the CNT-PVA composite (red line) and the pristine CNTs (black line). The
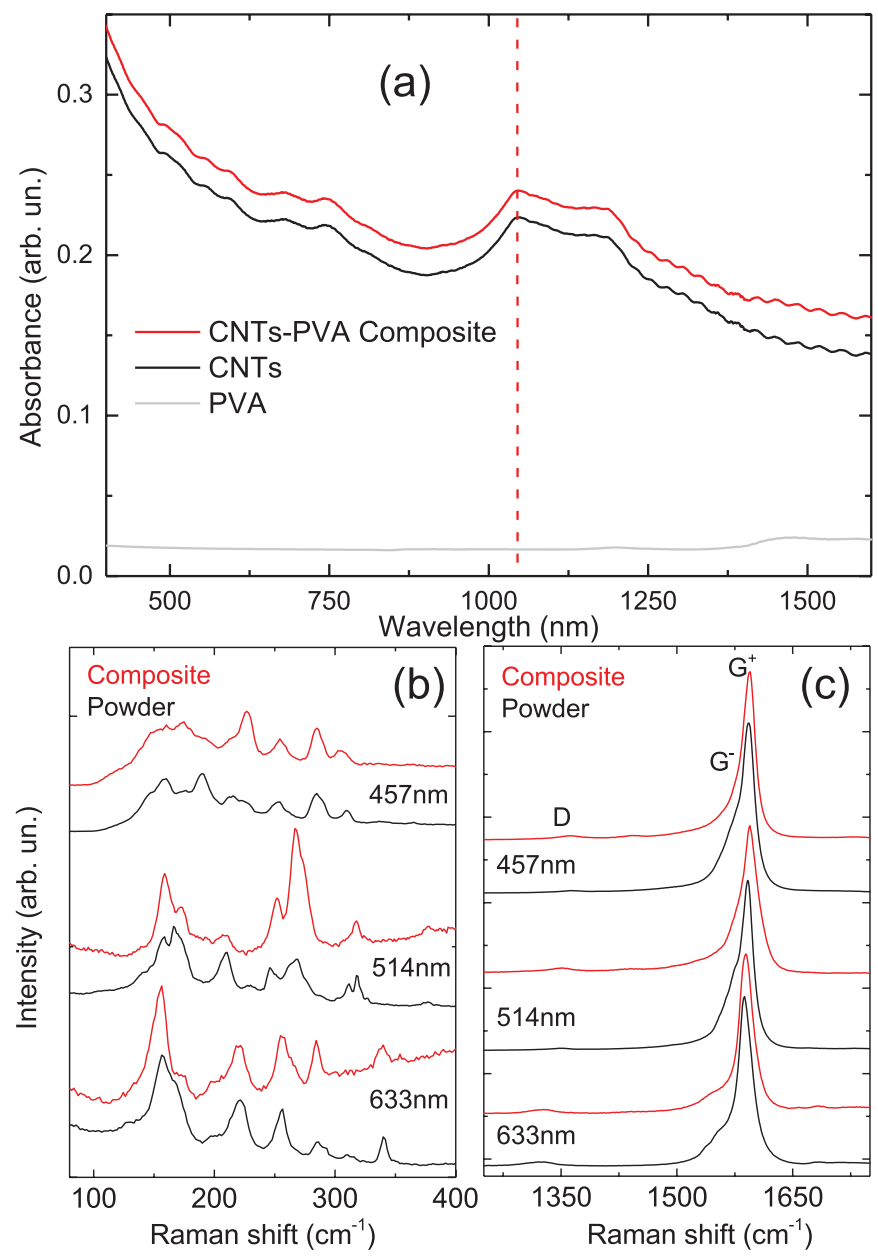

Fig. 1. (a) Absorption spectrum of CNTs (black line), CNT-PVA composite (red line), and PVA (grey line). The operating wavelength is marked by the red dashed line. Raman spectra of CNT powders (black lines) and CNT-PVA composite (red lines) at different excitation wavelengths: (b) RBM region and (c) $\mathrm{G}$ region.

absorption of the PVA is $\sim 1$ order of magnitude lower with respect to the CNT-PVA composite in the $400-1600 \mathrm{~nm}$ range and thus, is negligible.

For our laser, we use an all-fiber ring cavity design (Fig. 2). The active fiber is $0.6 \mathrm{~m}$ PM double-clad ytterbiumdoped fiber, pumped by a laser diode (LD) at $962 \mathrm{~nm}$. Wavelength division multiplexers (WDMs) couple pump light into the cavity and extract undepleted pump light. The CNT-SA is then integrated into the cavity by sandwiching the composite between two fiber connectors for angled physical contact (FC-APC). A PM isolator ensures unidirectional operation and a PM output coupler (OC) provides a 33\% output. A $300 \mathrm{~m}$ length of PM highly nonlinear fiber (HNLF; group velocity dispersion $\sim 24 \mathrm{ps}^{2} / \mathrm{km}$ ) is used to elongate the cavity and increase the total intracavity dispersion and nonlinearity.

The remainder of the cavity consists of PM980 Panda fiber. HNLF is spliced to PM980 fiber with $\sim 0.3 \mathrm{~dB}$ splice loss and $>30 \mathrm{~dB}$ polarization extinction ratio. The total cavity length is $\sim 312 \mathrm{~m}$.

\section{RESULTS AND DISCUSSION}

The laser exhibits self-starting mode-locking, producing a train of pulses with a $1.56 \mu$ s period, defined by the fundamental cavity frequency of $641 \mathrm{kHz}$ [Fig. 3(a) inset]. The pulse 


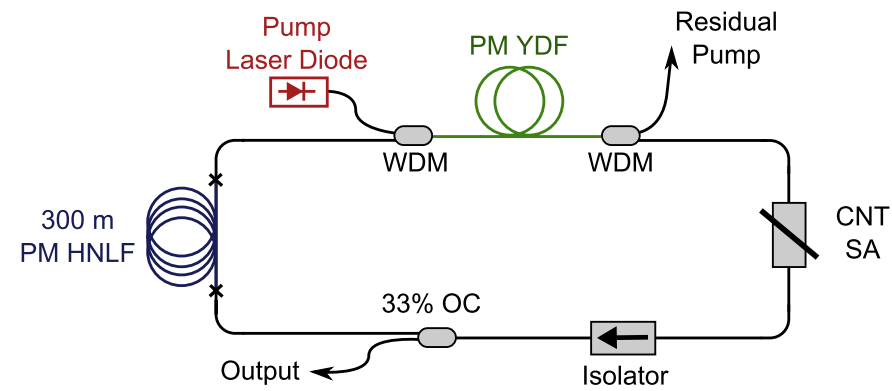

Fig. 2. Schematic setup of laser cavity: YDF - ytterbium-doped fiber; WDM - wavelength division multiplexer; CNT SA - nanotube-based saturable absorber; OC - output coupler; HNLF - highly nonlinear fiber.
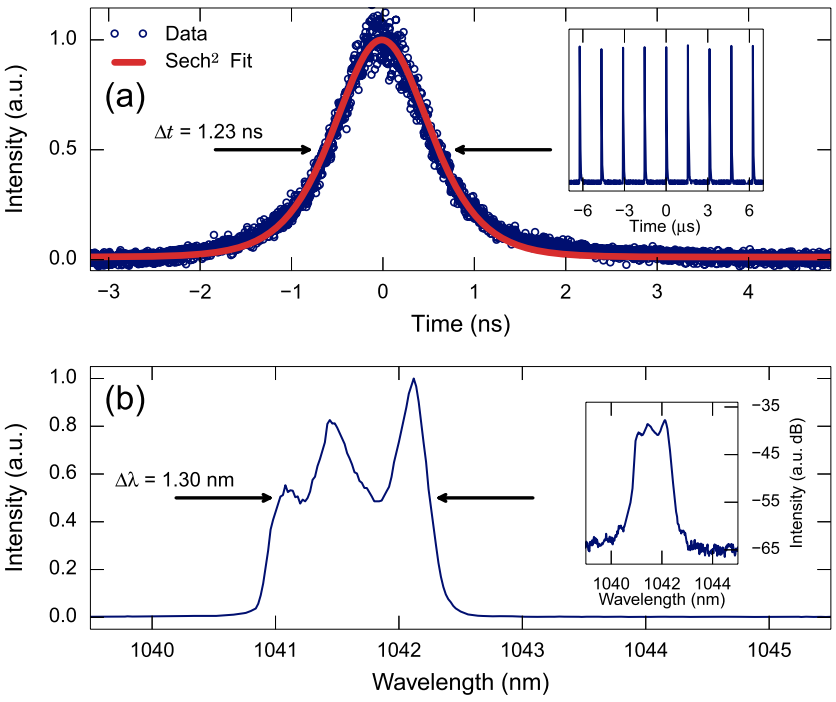

Fig. 3. Characteristics of laser output. (a) Streak camera trace (inset: pulse train on oscilloscope). (b) Optical spectrum on linear scale (inset: log scale).

energy is $0.20 \mathrm{~nJ}$. Operational characteristics are unchanged when the system is intentionally perturbed by mechanically stressing the cavity fiber, demonstrating the robust modelocking performance and environmental stability of the laser.

Temporal output properties are measured using a streak camera, showing the pulse full width at half maximum (FWHM) is $1.23 \mathrm{~ns}$ [Fig. 3(a)]. This is, to our knowledge, the longest duration reported to date for mode-locked pulses from an all-PM fiber laser. The data are well fit by a sech ${ }^{2}$ profile, as expected for pulses in long-cavity all-normal dispersion lasers [5]. Fig. 3(b) shows the corresponding output spectrum, which is centered at $1041.7 \mathrm{~nm}$ with a FWHM of $1.3 \mathrm{~nm}$. The steep spectral edges agree with the expected spectral shape for all-normal dispersion lasers producing dissipative solitons [8]. We also observed no noise-like structures when monitoring the output with a 15 ps photodiode, confirming that the laser is not operating in a noise-burst regime. These pulse properties correspond to a time-bandwidth product (TBP) of 442, a thousand times greater than the transform-limited TBP of 0.315 [16], suggesting that the pulse is significantly chirped. Also, unlike many reported all-normal dispersion lasers [8], [10], [11], we do not require an additional spectral filter to stabilize modelocking. Instead, the limited gain bandwidth of the system, including a spectral filtering effect upon highly chirped pulses from the CNT-SA [6], could provide a stabilizing effect.

Compared to passively mode-locked nanosecond pulse sources reported to date [3]-[6], our measured spectral width
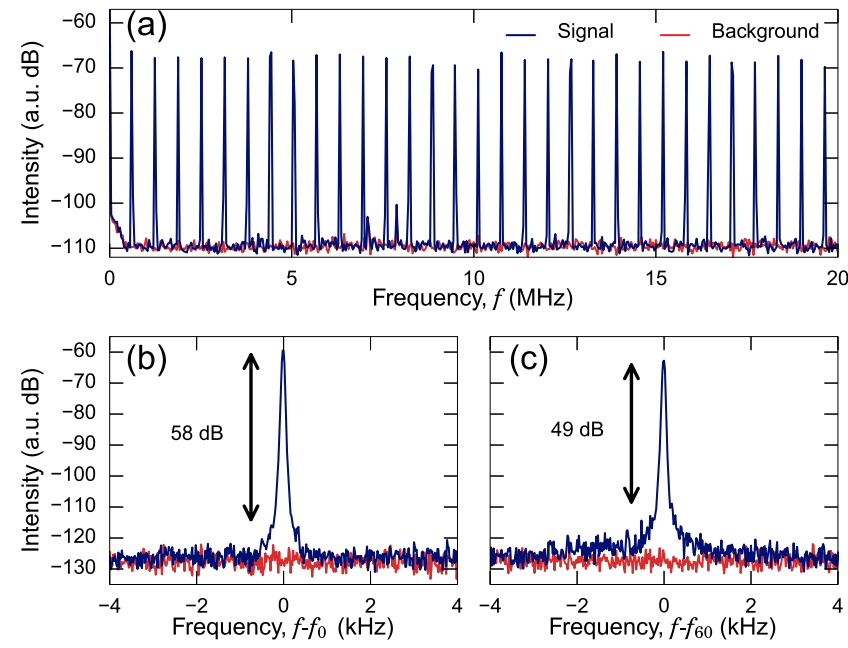

Fig. 4. RF spectrum of laser output: (a) $20 \mathrm{MHz}$ span. (b) $8 \mathrm{kHz}$ span about fundamental frequency, $f_{0}=641 \mathrm{kHz}$. (c) $8 \mathrm{kHz}$ span about $60^{\text {th }}$ harmonic, $f_{60}=38.46 \mathrm{MHz}(30 \mathrm{~Hz}$ resolution bandwidth).

of $1.3 \mathrm{~nm}$ is broader by more than a factor of two, despite using a much shorter cavity length. We attribute this to our use of highly nonlinear fiber to extend the cavity, rather than standard silica fiber. This increases the total cavity nonlinearity, so pulses experience greater self-phase modulation (SPM) which causes spectral broadening. Spectral broadening is then coupled to temporal broadening by the fiber group velocity dispersion. Therefore, by using HNLF we can generate nanosecond pulses with shorter cavity lengths than previously reported [3]-[6]. We suggest that adjusting cavity nonlinearity may be an alternative route to manipulating the pulse duration of all-normal dispersion lasers, preventing the need for ultralong cavities or the fabrication of chirped fiber Bragg gratings to provide large dispersion. This also highlights the need for further work to understand the complex pulse dynamics in the giant-chirped pulse regime.

The polarization of our chirped pulses is monitored using a home-made polarimeter. This consists of an optical power meter placed behind a quarter wave-plate and linear polarizer (in addition to collimating optics), both of which can be rotated so that Stokes parameters can be measured. From this, we calculate a DOP of $97 \%$, with a small ellipticity of 3 degrees. The extinction between the two polarization axes is $14.1 \mathrm{~dB}$. This confirms that the output is linearly-polarized, indicating scalar pulse generation.

All previously reported nanosecond-duration mode-locked pulse sources employ a non-PM cavity [3]-[6], with a polarization controller tuned to achieve a stable mode-locking state [4], [5]. Our all-PM cavity eliminates this requirement and mode-locking is always self-starting with no need for tuning.

Stability is probed using the methods outlined in [44] by recording the RF spectrum using a $1 \mathrm{GHz}$ photodetector and RF spectrum analyzer. The $20 \mathrm{MHz}$ span plot shows a steady train of cavity harmonics without spectral modulation [Fig. 4(a)], indicating no Q-switching instabilities [44]. We observe a high signal-to-background ratio of $58 \mathrm{~dB}$ $\left(10^{5.8}\right.$ contrast $)$ for the fundamental [Fig. 4(b)], which suggests good pulse-train stability. A low-level pedestal with a peak at $\sim-115 \mathrm{~dB}$ is also detected, from which the pulse-to-pulse 
energy fluctuations can be estimated [44]: $\frac{\Delta E}{E}=\left(\frac{\Delta P \Delta f}{\Delta f_{\text {rbw }}}\right)^{0.5}$ where $\Delta P$ is the pedestal-peak to signal-peak power ratio, $\Delta f$ is the width of the pedestal and $\Delta f_{\mathrm{rbw}}$ is the resolution of the spectrum analyzer for this measurement. With $\Delta P=$ $10^{-5.8}, \Delta f=500 \mathrm{~Hz}$ and $\Delta f_{\mathrm{rbw}}=30 \mathrm{~Hz}$, the energy fluctuation is $0.5 \%$. This is a low pulse energy fluctuation, especially compared to other nanosecond pulse sources, such as the $7 \%$ fluctuation reported in [14]. The $60^{\text {th }}$ harmonic can also be seen to exhibit a high $49 \mathrm{~dB}$ peak-to-background ratio [Fig. 4(c)], further confirming the mode-locking stability.

\section{COnclusions}

We reported the generation of scalar nanosecond-duration pulses from an environmentally stable mode-locked laser. The use of PM fiber throughout the cavity results in robust, selfstarting mode-locking, resistant to external disturbances. This paves the way to the development of mode-locked lasers generating scalar, linearly-polarized pulses with nanosecond durations and hundreds of kilohertz repetition rates for applications where reliability and stability are key requirements.

\section{REFERENCES}

[1] M. E. Fermann and I. Hartl, "Ultrafast fibre lasers," Nature Photon., vol. 7, pp. 868-874, Oct. 2013.

[2] W. H. Renninger, A. Chong, and F. W. Wise, "Giant-chirp oscillators for short-pulse fiber amplifiers," Opt. Lett., vol. 33, no. 24, pp. 3025-3027, 2008.

[3] S. Kobtsev, S. Kukarin, and Y. Fedotov, "Ultra-low repetition rate mode-locked fiber laser with high-energy pulses," Opt. Exp., vol. 16, no. 26, pp. 21936-21941, 2008.

[4] E. J. R. Kelleher et al., "Nanosecond-pulse fiber lasers mode-locked with nanotubes," Appl. Phys. Lett., vol. 95, no. 11, p. 111108, 2009.

[5] E. J. R. Kelleher et al., "Generation and direct measurement of giant chirp in a passively mode-locked laser," Opt. Lett., vol. 34, no. 22, pp. 3526-3528, 2009.

[6] X. Tian, M. Tang, X. Cheng, P. P. Shum, Y. Gong, and C. Lin, "High-energy wave-breaking-free pulse from all-fiber mode-locked laser system," Opt. Exp., vol. 17, no. 9, pp. 7222-7227, 2009.

[7] R. I. Woodward et al., "Nanotube mode-locked, low repetition rate pulse source for fiber-based supercontinuum generation at low average pump power," in Proc. Conf. Lasers Electro-Opt., Jun. 2014, paper STh3N.8.

[8] W. H. Renninger et al., "Dissipative solitons in normal-dispersion fiber lasers," Phys. Rev. A, vol. 77, no. 2, p. 023814, 2008.

[9] P. Grelu and N. Akhmediev, "Dissipative solitons for mode-locked lasers," Nature Photon., vol. 6, pp. 84-92, Feb. 2012.

[10] A. Chong, J. Buckley, W. Renninger, and F. Wise, "All-normaldispersion femtosecond fiber laser," Opt. Exp., vol. 14, no. 21, pp. 10095-10100, 2006.

[11] S. Lefrançois, K. Kieu, Y. Deng, J. D. Kafka, and F. W. Wise, "Scaling of dissipative soliton fiber lasers to megawatt peak powers by use of large-area photonic crystal fiber," Opt. Lett., vol. 35, no. 10, pp. $1569-1571,2010$.

[12] C. J. S. de Matos et al., "All-fiber format compression of frequency chirped pulses in air-guiding photonic crystal fibers," Phys. Rev. Lett., vol. 93, no. 10, p. 103901, 2004

[13] M. Zhang, E. J. R. Kelleher, E. D. Obraztsova, S. V. Popov, and J. R. Taylor, "Nanosecond pulse generation in lumped normally dispersive all-fiber mode-locked laser," IEEE Photon. Technol. Lett., vol. 23, no. 19, pp. 1379-1381, Oct. 1, 2011.

[14] L. Zhang, A. R. El-Damak, Y. Feng, and X. Gu "Experimental and numerical studies of mode-locked fiber laser with large normal and anomalous dispersion," Opt. Exp., vol. 21, no. 10, pp. 12014-12021, 2013.

[15] J. M. Dudley and J. R. Taylor, Supercontinuum Generation in Optical Fibers. Cambridge, U.K.: Cambridge Univ. Press, 2010.

[16] G. P. Agrawal, Nonlinear Fiber Optics. New York, NY, USA: Academic, 2001.

[17] S. T. Cundiff, B. C. Collings, N. N. Akhmediev, J. M. Soto-Crespo, K. Bergman, and W. H. Knox, "Observation of polarization-locked vector solitons in an optical fiber," Phys. Rev. Lett., vol. 82, no. 20, pp. 3988-3991, 1999
[18] J. H. Wong et al., "Vector solitons in a laser passively mode-locked by single-wall carbon nanotubes," Opt. Commun., vol. 284, no. 7, pp. 2007-2011, 2011

[19] X. Liu, J. Lægsgaard, and D. Turchinovich, "Monolithic highly stable $\mathrm{Yb}$-doped femtosecond fiber lasers for applications in practical biophotonics," IEEE J. Sel. Topics Quantum Electron., vol. 18, no. 4, pp. 1439-1450, Jul./Aug. 2012.

[20] C. Hnatovsky, V. G. Shvedov, and W. Krolikowski, "The role of light-induced nanostructures in femtosecond laser micromachining with vector and scalar pulses," Opt. Exp., vol. 21, no. 10, pp. 12651-12656, 2013.

[21] M. Erkintalo, C. Aguergaray, A. Runge, and N. G. R. Broderick, "Environmentally stable all-PM all-fiber giant chirp oscillator," Opt. Exp., vol. 20, no. 20, pp. 22669-22674, 2012.

[22] M. Baumgartl, J. Abreu-Afonso, A. Díez, M. Rothhardt, J. Limpert, and A. Tünnermann, "Environmentally stable picosecond $\mathrm{Yb}$ fiber laser with low repetition rate," Appl. Phys. B, vol. 111, no. 1, pp. 39-43, 2012.

[23] V. Scardaci et al., "Carbon nanotube polycarbonate composites for ultrafast lasers," Adv. Mater, vol. 20, no. 21, pp. 4040-4043, 2008

[24] T. Hasan et al., "Nanotube-polymer composites for ultrafast photonics," Adv. Mater., vol. 21, nos. 38-39, pp. 3874-3899, 2009.

[25] Z. Sun et al., "Graphene mode-locked ultrafast laser," ACS Nano, vol. 4, no. 2, pp. 803-810, 2010 .

[26] J. S. Lauret et al., "Ultrafast carrier dynamics in single-wall carbon nanotubes," Phys. Rev. Lett. vol. 90, no. 5, p. 057404, 2003.

[27] D. Brida et al., "Ultrafast collinear scattering and carrier multiplication in graphene," Nature Commun., vol. 4, p. 1987, Jun. 2013.

[28] D. Popa, Z. Sun, F. Torrisi, T. Hasan, F. Wang, and A. C. Ferrari, "Sub $200 \mathrm{fs}$ pulse generation from a graphene mode-locked fiber laser," Appl. Phys. Lett., vol. 97, no. 20, p. 203106, 2010.

[29] D. Popa et al., "74-fs nanotube-mode-locked fiber laser," Appl. Phys. Lett., vol. 101, no. 15, p. 153107, 2012.

[30] F. Wang et al., "Wideband-tuneable, nanotube mode-locked, fibre laser," Nature Nanotechnol., vol. 3, pp. 738-742, Nov. 2008.

[31] M. Zhang et al., "Tm-doped fiber laser mode-locked by graphenepolymer composite," Opt. Exp., vol. 20, no. 22, pp. 25077-25084, 2012.

[32] F. Bonaccorso, Z. Sun, T. Hasan, and A. C. Ferrari, "Graphene photonics and optoelectronics," Nature Photon., vol. 4, pp. 611-622, Aug. 2010.

[33] Z. Sun, T. Hasan, and A. C. Ferrari, "Ultrafast lasers mode-locked by nanotubes and graphene," Phys. E, Low-Dimensional Syst. Nanostruct., vol. 44, no. 6, pp. 1082-1091, 2012.

[34] C. A. Zaugg et al., "Ultrafast and widely tuneable vertical-externalcavity surface-emitting laser, mode-locked by a graphene-integrated distributed Bragg reflector," Opt. Exp., vol. 21, no. 25, pp. 31548-31559, 2013.

[35] R. B. Weisman and S. M. Bachilo "Dependence of optical transition energies on structure for single-walled carbon nanotubes in aqueous suspension: An empirical Kataura plot," Nano Lett., vol. 3, no. 9, pp. 1235-1238, 2003.

[36] K. Liu et al., "An atlas of carbon nanotube optical transitions," Nature Nanotechnol. vol. 7, pp. 325-329, 2012.

[37] E. Flahaut, C. Laurent, and A. Peigney, "Catalytic CVD synthesis of double and triple-walled carbon nanotubes by the control of the catalyst preparation," Carbon, vol. 43, no. 2, pp. 375-383, 2005.

[38] H. Telg, J. Maultzsch, S. Reich, F. Hennrich, and C. Thomsen, "Chirality distribution and transition energies of carbon nanotubes," Phys. Rev. Lett., vol 93, no. 17, p. 177401, 2004.

[39] C. Fantini, A. Jorio, M. Souza, M. S. Strano, M. S. Dresselhaus, and M. A. Pimenta, "Optical transition energies for carbon nanotubes from resonant Raman spectroscopy: Environment and temperature effects," Phys. Rev. Lett., vol. 93, no. 14, p. 147406, 2004.

[40] J. C. Meyer et al., "Raman modes of index-identified freestanding single-walled carbon nanotubes," Phys. Rev. Lett., vol. 95, no. 21, p. 217401, 2005.

[41] F. Bonaccorso et al., "Density gradient ultracentrifugation of nanotubes: Interplay of bundling and surfactants encapsulation," J. Phys. Chem. C, vol. 114 , no. 41, pp. 17267-17285, 2010.

[42] A. C. Ferrari and J. Robertson, "Interpretation of Raman spectra of disordered and amorphous carbon," Phys. Rev. B vol. 61, no. 20, p. $14095,2000$.

[43] A. C. Ferrari and D. M. Basko, "Raman spectroscopy as a versatile tool for studying the properties of graphene," Nature Nanotechnol., vol. 8, pp. 235-246, Apr. 2013.

[44] D. Linde, "Characterization of the noise in continuously operating mode-locked lasers," Appl. Phys. B, vol. 39, no. 4, pp. 201-217, 1986. 